\title{
Is the Strongest Level of Medical Evidence Always Required for Guidelines Recommendations?
}

\author{
Matteo Ravaioli ${ }^{a}$ b Fabio Piscagliaa, ${ }^{a}$ Umberto Cillo ${ }^{d}$ Giovanni Brandi ${ }^{e, f}$ \\ Maurizio Sessag ${ }^{9}$ Giuliana Germinario ${ }^{b}$ Rita Golfierie, h Alberta Cappelli ${ }^{\text {h }}$ \\ Maria C. Morellib Antonio Siniscalchi ${ }^{i}$ Antonietta D'Errico ${ }^{e, j}$ Matteo Cescon $^{a, b}$ \\ Primiano lannone $^{k}$ Franco Trevisani $^{a}{ }^{\text {I }}$

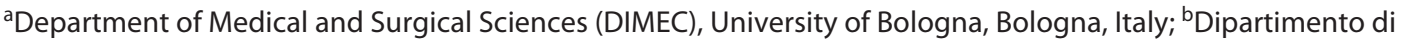 \\ Chirurgia Generale e Trapianti, IRCCS, Azienda Ospedaliero-Universitaria di Bologna, Bologna, Italy; ' $D$ Division of \\ Internal Medicine, Department of Digestive Disease and Internal Medicine, IRCCS, Azienda Ospedaliero-Universitaria \\ di Bologna, Bologna, Italy; ${ }^{d}$ Hepatobiliary Surgery and Liver Transplant Unit, University of Padua, Padua, Italy; \\ eDepartment of Experimental, Diagnostic, and Specialty Medicine, University of Bologna, Bologna, Italy; ${ }^{f}$ Division \\ of Oncology, IRCCS, Azienda Ospedaliero-Universitaria di Bologna, Bologna, Italy; ${ }^{9}$ Department of Drug Design

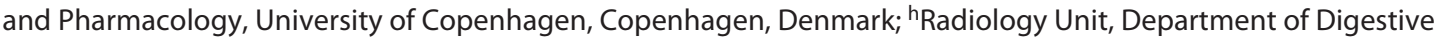 \\ Disease and Internal Medicine, IRCCS, Azienda Ospedaliero-Universitaria di Bologna, Bologna, Italy; 'Division of \\ Anesthesiology, Department of Anesthesia and Intensive Care, IRCCS, Azienda Ospedaliero-Universitaria di Bologna,

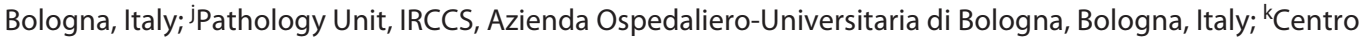 \\ Eccellenza Clinica, Qualità e Sicurezza delle Cure, Istituto Superiore di Sanità, Rome, Italy; 'Semeiotica Medica Unit, \\ IRCCS, Azienda Ospedaliero-Universitaria di Bologna, Bologna, Italy
}

Dear Editor,

We read with interest the randomized multicentric study of liver transplantation (LT) for patients with downstaged hepatocellular carcinoma (HCC), recently published in the Lancet Oncology Journal [1]. The authors were able to demonstrate the greater efficacy of LT over the alternative nonsurgical standard of care therapy, according to the strongest level of medical evidence. We believe the present study is an important occasion to discuss the relevant issue of how to introduce in clinical practice new indications in the setting of LT.

Clinical recommendations are formulated according to the relevant evidence, and a new surgical strategy may take up to several years to be fully validated before its introduction in clinical practice. Phase 3 randomized trials are the required ultimate evidence to produce updates of guideline recommendations, but they are often extremely difficult to be completed in the surgical setting. Conse-

karger@karger.com www.karger.com/lic

Karger:
(C) 2021 The Author(s)

Published by S. Karger AG, Basel

This is an Open Access article licensed under the Creative Commons Attribution-NonCommercial-4.0 International License (CC BY-NC) (http://www.karger.com/Services/OpenAccessLicense), applicable to the online version of the article only. Usage and distribution for commercial purposes requires written permission. quently, the authors of the article are to be commended for their effort to produce the highest level of evidence to confirm the survival benefit of LT in downstaged HCC. But will these efforts remain always necessary?

Some authors consider that a difference in outcome greater than $25 \%$ based on preliminary studies is a reason for not proceeding to studies including randomization [2]. A randomized trial aimed at confirming a potential benefit $>25 \%$ may place physicians in a very uncomfortable position of choosing between the already expected best strategy for the patient and that required by the rules of the strength of evidence.

Mazzaferro et al.'s [1] trial was designed in 2010 and enrolled 74 patients between 2011 and 2015. Forty-three patients who achieved successful downstaging were randomized: the study arm (21 cases) received LT experiencing no dropout, also thanks to a relatively short waiting time, while the control arm (22 cases) continued the fol- 
low-up until tumor progression, when surgical, locoregional, or systemic therapies were applied.

The article showed a significantly better patient survival in the transplanted arm (5-year survival rate: $77.5 \mathrm{vs.}$ $31.2 \%)$, confirming the results of previous prospective cohort studies $[3,4]$. The results of these cohort studies convinced many centers (including those of Bologna and $\mathrm{Pa}$ dova) to start performing LT in successfully downstaged HCC patients on a regular basis, and this conflicted with the participation of such centers in a randomized study.

However, according to the currently required levels of evidence, we should wonder whether these centers adopted in clinical practice a correct behavior even if not supported by randomized studies. We agree that the previous level of evidence for this strategy was lower than that provided by the present study [1], but we must also consider that accepting the lower level of evidence provided by cohort studies, many patients have been correctly treated over these years.

We did not want to compare the cohort to randomized studies, but we aimed to focus on the methodology to produce clinical recommendations. The GRADE methodology (www.gradeworkinggroup.org), which is the standard adopted worldwide as the most reliable way to produce trustworthy clinical guidelines, acknowledges that randomized trials are neither sufficient nor necessary to produce strong recommendations when some other conditions are met as was the case of LT versus non-LT treatments for HCC. Also, special circumstances warrant strong recommendations when evidence is weak or conflicting [5].

We have great esteem for all the authors of the study, some of them being pioneers of LT. The aim of our comment was not to criticize the study design or its ethical implications but to open the discussion concerning the validity of large prospective cohort studies to change the clinical practice without the need of randomized trials.
Several other cohort studies [6] show that LT is superior to nontransplant therapies even for patients who did not fully complete the downstaging procedure or failed to reach the Milan criteria, provided that macrovascular invasion and extrahepatic spread of the tumor were absent. Should we actually need another randomized trial to accept to transplant these patients, an option which might never come again or, instead, should we make maximal efforts to establish how to design prospective cohort studies able to provide a level of evidence sufficient to make randomized trials unnecessary?

\section{Statement of Ethics}

Demographic, clinical, laboratory, and imaging data of study patients were collected and analyzed in accordance to the Helsinki Declaration and Regional Ethic Committee under informed consent of the patients.

\section{Conflict of Interest Statement}

Fabio Piscaglia is an editorial board member of Liver Cancer. The other authors have no conflicts of interest to declare.

\section{Funding Sources}

Research program among region and university.

\section{Author Contributions}

Conceptualization: M.R., F.P., U.C., G.B., M.C., and F.T.; acquisition of data: M.R., F.P., U.C., G.B., M.S., G.G., R.G., A.C., M.C.M., A.S., A.D.E., M.C., and F.T.; statistical analysis: M.S.; literature search: G.G., R.G., A.C., M.C.M., A.S., and A.D.E.; writing/ reviewing/editing: M.R., F.P., U.C., G.B., M.S., G.G., R.G., A.C., M.C.M., A.S., A.D.E., M.C., and F.T.

\section{References}

1 Mazzaferro V, Citterio D, Bhoori S, Bongini M, Miceli R, De Carlis L, et al. Liver transplantation in hepatocellular carcinoma after tumour downstaging (XXL): a randomised, controlled, phase $2 \mathrm{~b} / 3$ trial. Lancet Oncol. 2020;21(7):947-56.

2 Gehan EA, Freireich EJ. Non-randomized controls in cancer clinical trials. N Engl J Med. 1974;290(4):198-203.
3 Ravaioli M, Grazi GL, Piscaglia F, Trevisani F, Cescon M, Ercolani G, et al. Liver transplantation for hepatocellular carcinoma: results of down-staging in patients initially outside the Milan selection criteria. Am J Transplant. 2008;8(12):2547-57.

4 Yao FY, Kerlan RK Jr, Hirose R, Davern TJ 3rd, Bass NM, Feng S, et al. Excellent outcome following down-staging of hepatocellular carcinoma prior to liver transplantation: an intention-to-treat analysis. Hepatology. 2008; 48(3):819-27.
5 Andrews JC, Schünemann HJ, Oxman AD, Pottie K, Meerpohl JJ, Coello PA, et al. GRADE guidelines: 15. Going from evidence to recommendation-determinants of a recommendation's direction and strength. J Clin Epidemiol. 2013;66(7):726-35.

6 Ravaioli M, Odaldi F, Cucchetti A, Trevisani F, Piscaglia F, De Pace V, et al. Long term results of down-staging and liver transplantation for patients with hepatocellular carcinoma beyond the conventional criteria. Sci Rep. 2019;9(1):3781.
Liver Transplantation for Downstaged Hepatocellular Carcinoma
Liver Cancer 2021;10:394-395

DOI: $10.1159 / 000516196$ 